\title{
CycleGAN without checkerboard artifacts for counter-forensics of fake-image detection
}

\author{
Takayuki Osakabe $^{\mathrm{a}}$, Miki Tanaka ${ }^{\mathrm{b}}$, Yuma Kinoshita ${ }^{\mathrm{c}}$, and Hitoshi Kiya ${ }^{\mathrm{d}}$ \\ a-d Tokyo Metropolitan University, Tokyo, Japan
}

\begin{abstract}
In this paper, we propose a novel CycleGAN without checkerboard artifacts for counter-forensics of fake-image detection. Recent rapid advances in image manipulation tools and deep image synthesis techniques, such as Generative Adversarial Networks (GANs) have easily generated fake images, so detecting manipulated images has become an urgent issue. Most state-of-the-art forgery detection methods assume that images include checkerboard artifacts which are generated by using DNNs. Accordingly, we propose a novel CycleGAN without any checkerboard artifacts for counter-forensics of fake-mage detection methods for the first time, as an example of GANs without checkerboard artifacts.
\end{abstract}

Keywords: GAN, checkerboard artifacts, counter-forensics, deep learning

\section{INTRODUCTION}

Although deep neural networks (DNNs) have led to major breakthroughs in computer vision, for a wide range of applications, they have created new concerns and problems. DNNs in general suffer from attacks such as invasion of data privacy, ${ }^{1-3}$ and adversarial attacks. ${ }^{4-6}$ In addition, recent rapid advances in deep image synthesis techniques, such as Generative Adversarial Networks (GANs) ${ }^{7}$ have easily generated fake images, so detecting manipulated images has become an urgent issue.

So far, a lot of researchers have investigated forgery detection methods, in which most state-of-the-art forgery detection methods assume that images include checkerboard artifacts which are generated by using DNNs. ${ }^{89}$ In contrast, checkerboard artifacts-free DNNs have been proposed by using a fixed convolutional layer, ${ }^{10-12}$ but the technique for avoiding the artifacts have never been applied to img2img GANs like CycleGAN. In this paper, we propose a CycleGAN without checkerboard artifacts for the first time, and moreover, the proposed CycleGAN is demonstrated to be effective for counter-forensics of fake image detection methods, as an example of GANs without checkerboard artifacts.

\section{RELATED WORK}

CycleGAN allows us to convert one image into another, called image to image conversion. Figure 1 shows an example of images generated by using CycleGAN. CycleGAN is a model for unsupervised learning of image to image translation using an adversarial network with cycle-consistency, as shown in Fig.2. As shown in the figure, CycleGAN has two generators: $G_{A B}$ that converts images from domain A to domain B, and vice versa, $G_{B A}$. Discriminator $D_{B}$ encourages $G_{A B}$ to translate A into outputs indistinguishable from domain B. In this paper, we refer to images generated by GANs as fake images. Many researchers have been working on detecting images generated by using GANs. ${ }^{8,9}$ In typical fake-image detection methods such as the method proposed by Zhang et al., ${ }^{8}$ fake-images are detected by finding checkerboard artifacts included in manipulated images. 


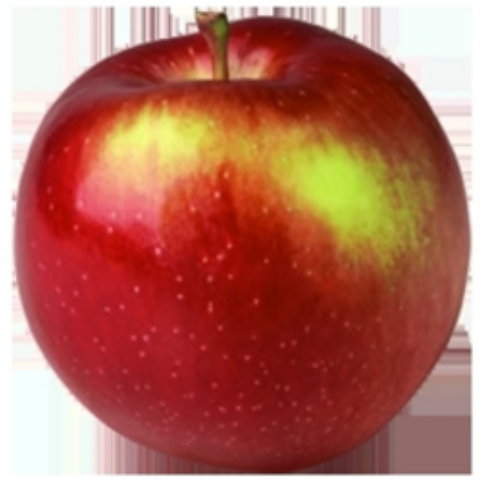

(a) real image

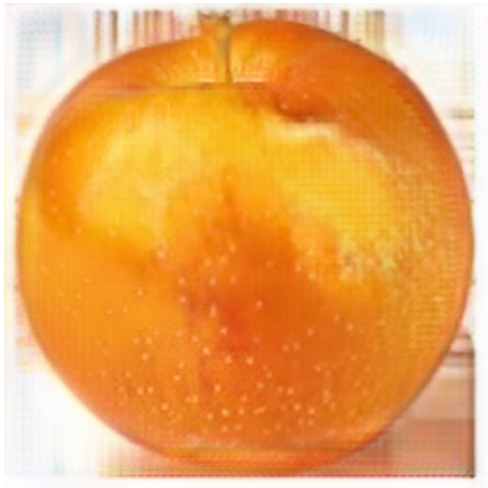

(b) fake image with artifacts

Figure 1: Example of fake images

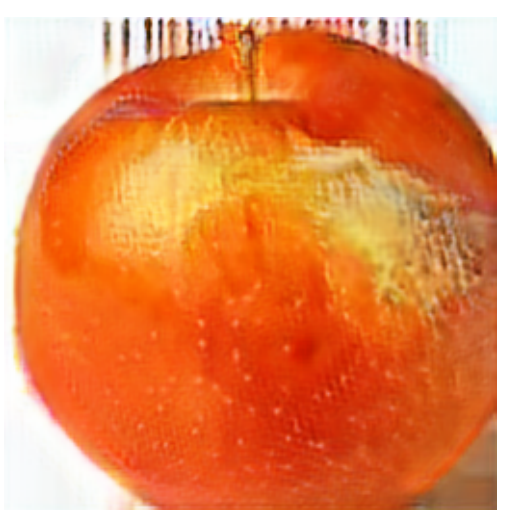

(c) fake image without artifacts

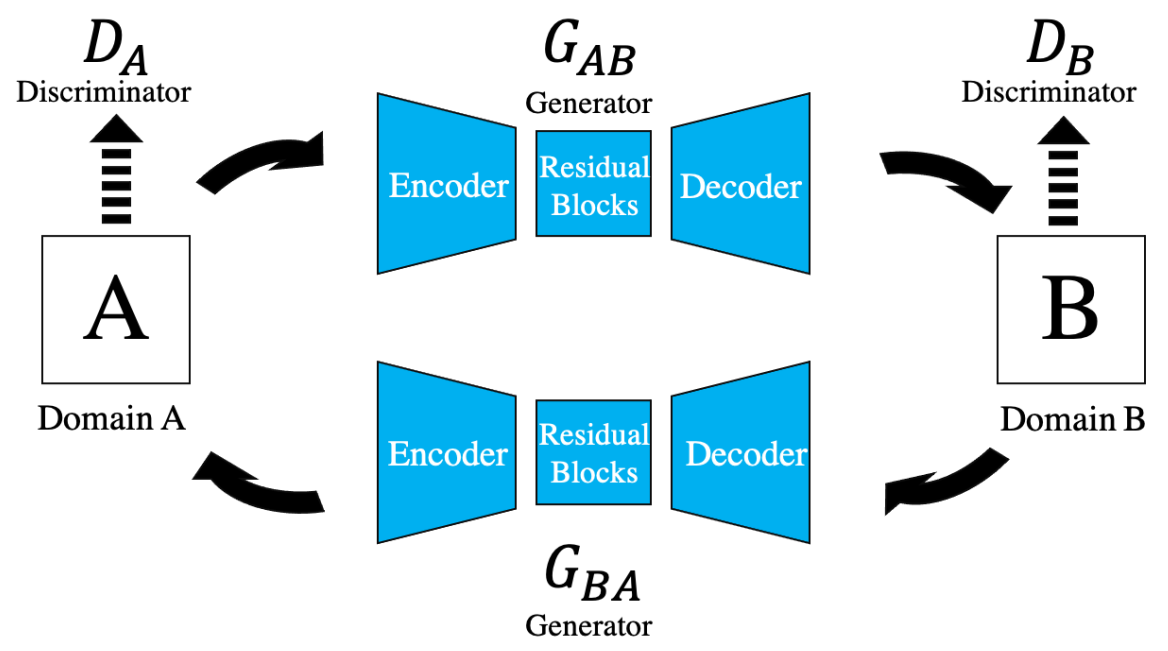

Figure 2: CycleGAN model

\section{CYCLE-GAN WITHOUT CHECKERBOARD ARTIFACTS}

Checkerboard artifacts are known to be caused in upsampling layers during forward propagation and in the downsampling layer during back propagation as in. ${ }^{10-12}$ By inserting a fixed convolution layer into upsampling and downsampling layers, the artifacts were demonstrated to be completely avoided, although this technique has not been applied to any image to image transformations like CyclcGAN. We have two aims in this paper. The first one is to apply this technique to CycleGAN to remove checkerboard artifacts included in fake images. The another is to confirm the effectiveness in counter-forensics against fake-image detection.

The structure of the generator of CycleGAN is shown in the Fig. $3 .^{13}$ In contrast, Fig. 4 shows the structure of the generator of the proposed CycleGAN without checkerboard artifacts, where fixed convolution layers are inserted in the encoder and decoder sections. In this paper, a fixed convolution layer is inserted into both every upsampling layer and every downsampling layer as in the paper. ${ }^{10}$ To constrain the Lipschitz constant, we also inserted Spectral Normalization layer ${ }^{14}$ into the discriminator and changed the Adversarial loss to Hinge loss.

Further author information:

T. Osakabe: E-mail: osakabe-takayuki@ed.tmu.ac.jp

M. Tanaka: E-mail: tanaka-miki@ed.tmu.ac.jp

Y. Kinoshita: E-mail: ykinoshita@tmu.ac.jp

H. Kiya: E-mail: kiya@tmu.ac.jp 


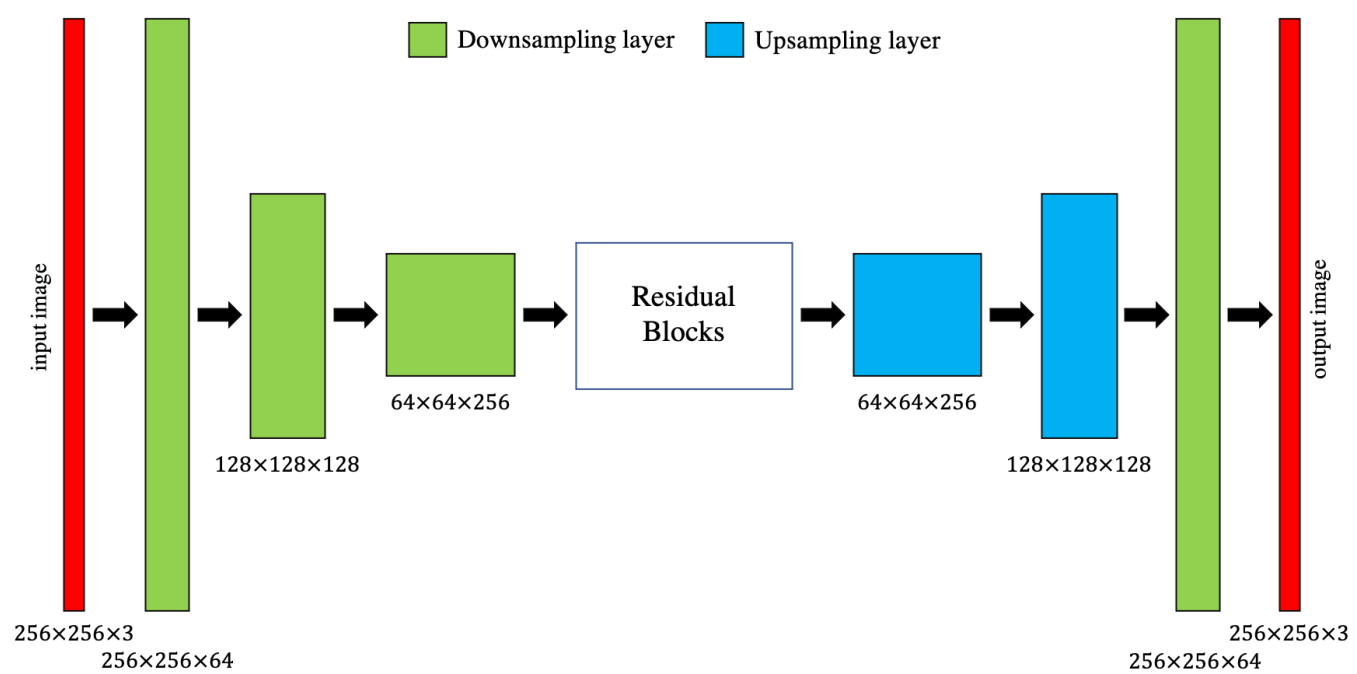

Figure 3: Generator of CycleGAN. Each box denotes multi-channel feature map produced by each layer. Output size of each layer is denoted under each box $($ height $\times$ width $\times$ channel $)$.

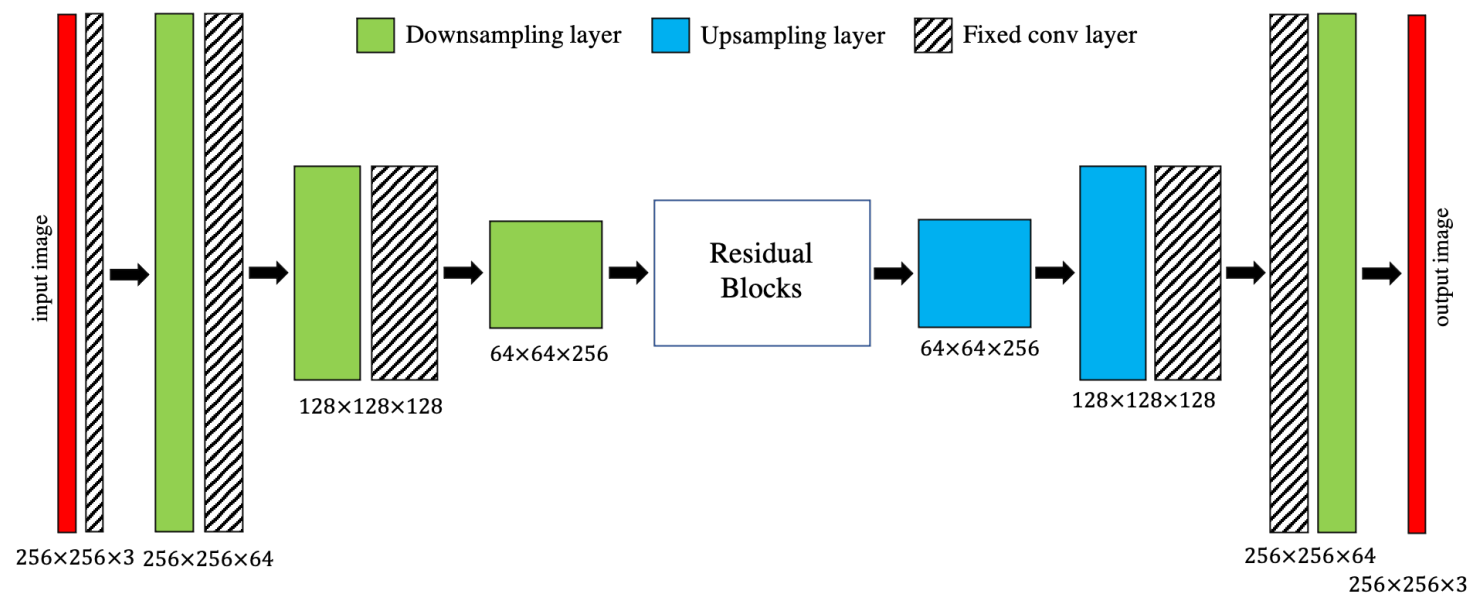

Figure 4: Generator of proposed CycleGAN without checkerboard artifacts. Each box denotes multi-channel feature map produced by each layer. Output size of each layer is denoted under each box $($ height $\times$ width $\times$ channel).

\section{EXPERIMENT}

In the experiment, CycleGAN models were trained under two conditions: with checkerboard artifacts (conventional) and without checkerboard artifacts (proposed). Fake images generated by using the models were applied to a fake-image detection method. ${ }^{8}$

\subsection{EXPERIMENTAL SETUP}

To train models, we used the same generator, discriminator structures and hyper parameters as those in. ${ }^{13}$ The apple2orange dataset was also used for training models and testing. ${ }^{13}$ This dataset consists of apple-images and orange-ones, so models were trained for designing an unpaired image-to-image transformation such as from apples to oranges.

\subsection{EXPERIMENTAL RESULTS}

Figure 1 shows an example of fake images generated by using the trained models, where the image in Fig.1 (a) is the input one (real). The image in Fig.1 (b) includes checkerboard artifacts, although it is not easy to visually find the artifacts. To clearly show the difference between two the fake images, the images were represented in the 


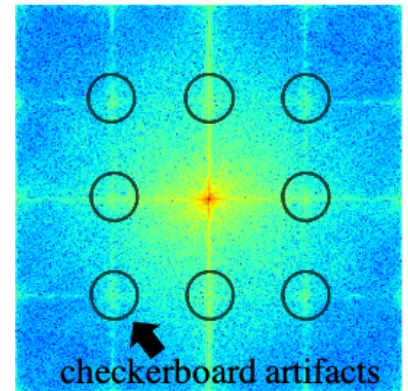

(a) Image in Fig.1(b)

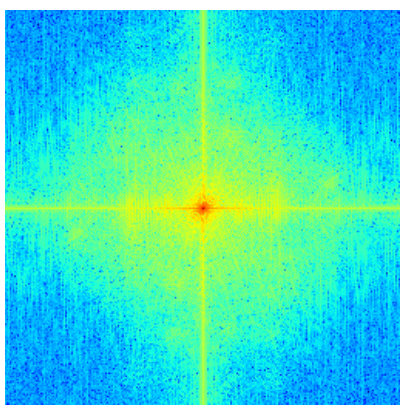

(b) Image in Fig.1(c)

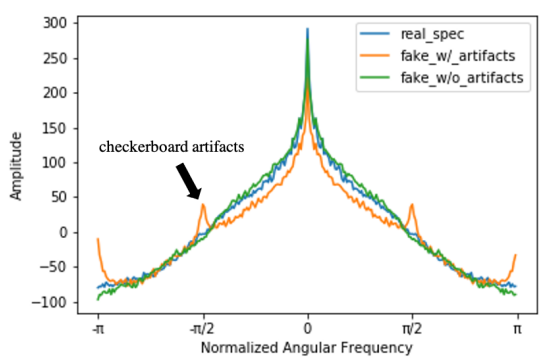

(c) Images in Fig.1(1D)

Figure 5: Spectrum of fake images

frequency domain as in Fig.5, where one-dimensional (1D) signals in the horizontal direction were also used to compute logarithmic spectrums as well as in the paper. ${ }^{8}$ From Fig.5, the proposed scheme was demonstrated to be effective in removing checkerboard artifacts even when GANs were applied to image to image transformation for generating fake images for the first time.

Next, the effectiveness of the proposed CycleGAN was evaluated in terms of detection accuracy under the use of a fake-image detection method. ${ }^{8}$ Experimental results are shown in Table 1, where w/o artifacts corresponds to the proposed method, and two accuracy indices: ACC and ACC (Fake) in Table 1 are defined by

$$
\begin{gathered}
A C C=\frac{N_{t n}+N_{t p}}{N_{Q f}+N_{Q r}} \\
A C C(\text { Fake })=\frac{N_{t n}}{N_{Q f}} .
\end{gathered}
$$

$N_{Q f}$ is the number of fake query images and $N_{Q r}$ is the number of real query ones in the data set. $N_{t n}$ also indicates the number of true negatives which are outcomes where the model correctly predicts the negative class, and $N_{t p}$ denotes the number of true positives which are outcomes which the model correctly predicts the positive class. From Table 1, the detection accuracy decreased when using the proposed GAN(w/o artifacts). This is because the detection method assumes that checkerboard artifacts are included in fake images. Accordingly, the proposed CyclicGAN was confirmed to be effective as a counter forensics method of fake-image detection.

Table 1: Comparison with conventional CycleGAN

\begin{tabular}{c|cccc}
\hline \multirow{2}{*}{ Dataset } & \multicolumn{2}{|c}{ w/ artifacts } & \multicolumn{2}{c}{ w/o artifacts (proposed) } \\
\cline { 2 - 5 } & ACC & ACC (Fake) & ACC & ACC (Fake) \\
\hline apple2orange & 0.85 & 0.92 & 0.46 & 0.12 \\
\hline
\end{tabular}

\section{CONCLUSION}

We proposed a novel CycleGAN without checkerboard artifacts for the first time, which allows us to reduce the detection accuracy of state-of-the-art fake-image detection methods. In the proposed CycleGAN, a fixed convolution layer is inserted into not only every upsampling layer but also every downsampling layer in generators. In the experiment, images generated by the proposed one were applied to a fake-image detection method so that the proposed method was also demonstrated to be effective in terms of detection accuracy of fake-images. 


\section{REFERENCES}

[1] Sirichotedumrong, W., Maekawa, T., Kinoshita, Y., and Kiya, H., "Privacy-preserving deep neural networks with pixel-based image encryption considering data augmentation in the encrypted domain," IEEE International Conference on Image Processing (2019).

[2] Sirichotedumrong, W. and Kiya, H., "Grayscale-based block scrambling image encryption using ycbcr color space for encryption-then-compression systems," APSIPA Trans. Signal and Information Processing 8, no.E7 (2019).

[3] Sirichotedumrong, W., Kinoshita, Y., and Kiya, H., "Pixel-based image encryption without key management for privacy-preserving deep neural networks," IEEE Access 7, 177844-177855 (2019).

[4] AprilPyone, M., Kinoshita, Y., and Kiya, H., "Adversarial robustness by one bit double quantization for visual classification," IEEE Access 7, 177932-177943 (2019).

[5] AprilPyone, M. and Kiya, H., "Block-wise image transformation with secret key for adversarially robust defense," arXiv:2010.00801 (2020).

[6] AprilPyone, M. and Kiya, H., "Encryption inspired adversarial defense for visual classification," IEEE International Conference on Image Processing (2020).

[7] Goodfellow, I. J., Pouget-Abadie, J., Mirza, M., Xu, B., Warde-Farley, D., Ozair, S., Courville, A., and Bengio, Y., "Generative adversarial nets," Neural Information Processing Systems (2014).

[8] Zhang, X., Karaman, S., and Chang, S.-F., "Detecting and simulating artifacts in gan fake images," IEEE International Workshop on Information Forensics and Security (2019).

[9] Wang, S.-Y., Wang, O., Zhang, R., Owens, A., and Efros, A. A., "Cnn-generated images are surprisingly easy to spot... for now," Computer Vision and Pattern Recognition, 8695-8704 (2020).

[10] Kinoshita, Y. and Kiya, H., "Fixed smooth convolutional layer for avoiding checkerboard artifacts in cnns," IEEE International Conference on Acoustics, Speech and Signal Processing, 3712-3716 (2020).

[11] Sugawara, Y., Shiota, S., and Kiya, H., "Checkerboard artifacts free convolutional neural networks," APSIPA Trans. Signal and Information Processing 8, no.E9 (2019).

[12] Sugawara, Y., Shiota, S., and Kiya, H., "Super-resolution using convolutional neural networks without any checkerboard artifacts," IEEE International Conference on Image Processing (2018).

[13] Zhu, J.-Y., Park, T., Isola, P., and Efros, A. A., "Unpaired image-to-imagetranslation using cycle-consistent adversarial networks," International Conference of Computer Vision (2017).

[14] Miyato, T., Kataoka, T., Koyama, M., and Yoshida, Y., "Spectral normalization for generative adversarial networks," International Conference on Learning Representations (2018). 